\title{
The Application of the in-Situ Hyperthermia Emission from Acoustic Nanodroplets for Theranostic Dual-Imaging and Antitumor Modalities
}

\author{
Rodi Abdalkader, ${ }^{a}$ Johan Unga, ${ }^{b}$ Kazuo Maruyama, ${ }^{b}$ Fumiyoshi Yamashita, ${ }^{c}$ and \\ Mitsuru Hashida*,a \\ ${ }^{a}$ Institute for Advanced Study (KUIAS), Institute for Integrated Cell-Material Sciences (iCeMS), Kyoto University; \\ Yoshida Ushinomiya, Sakyo-ku, Kyoto 606-8501, Japan: ${ }^{b}$ Faculty of Pharma-Sciences, Teikyo University; 2-11-1 \\ Kaga, Itabashi-ku, Tokyo 173-8605, Japan: and ${ }^{c}$ Graduate School of Pharmaceutical Sciences, Kyoto University; \\ 46-29 Yoshida-Shimo-Adachi, Sakyo-ku, Kyoto 606-8501, Japan.
}

Received February 27, 2020; accepted April 20, 2020; advance publication released online May 2, 2020

In this study, we have developed a theranostic nanocarrier that can emit heat upon the exposure to ultrasound (US) irradiation as well as the generation of a contrast signal that can be detected with ultrasonography. The prepared acoustic nanodroplets (NDs) made with liquid perfluporopentane (PFPn) had an average size of $197.7 \pm 3.6 \mathrm{~nm}$ in diameter and were stable in vitro for $60 \mathrm{~min}$. US irradiation at $2 \mathrm{~W} . \mathrm{cm}^{-2}$ induced phase change of NDs into bubbles in vitro. On the other hand, the intra-tumor injection of NDs in combination with US irradiation induced thermal emission in situ in B16BL6 melanoma tumor implanted into mice and the emission areas have mostly covered the tumor site. Also, the combination between NDs and US irradiation has inhibited the tumor growth. Under this condition, the heat shock protein (HSP70) in tumor was significantly upregulated after $6 \mathrm{~h}$ of the treatment of NDs with US. Thus, we have developed a therapeutic system with multiple theranostic modalities composed of acoustic NDs and US irradiation applicable to the tumor treatment on the external surface of the body.

Key words acoustic nanodroplet; ultrasound irradiation; contrast signal; thermal emission; tumor inhibition; heat shock protein

\section{INTRODUCTION}

In recent years, there has been an emerging trend for the development of new therapeutic and diagnostic platforms for cancer treatment; including the fusion system of cutting-edge nanomedicines and imaging modalities such as magnetic resonance imaging (MRI), positron emission tomography/single photon emission tomography (PET/SPECT), and ultrasonography. ${ }^{1-3)}$ These combinational modalities of therapy and diagnostics also known as "Theranostics" are attracting a huge attention. ${ }^{4)}$ For example, theranostics-based modalities have the capability in treating the tumor concurrently with an imaging platform for a quick feedback of the tumor prognosis. ${ }^{5)}$

We as well as other groups have previously showed the theranostic potential of ultrasound contrast agents such as microbubbles, ${ }^{6,7)}$ nanobubbles, ${ }^{8,9)}$ and acoustic nanodroplets. ${ }^{4,10)}$ Acoustic nanodroplets (NDs) have superior characteristics to other ultrasound contrast agents due to their small sizes and stability in vivo. We have reported the characteristics of theranostic perfluorohexane-based acoustic NDs made up with phospholipids polyethylene glycol (PEG) shells. ${ }^{11)}$ These NDs were stable for $60 \mathrm{~min}$ at the blood circulation in mice. Additionally, upon exposure to ultrasound irradiation, NDs could change from a liquid state into a gas state. In this transition, the gas formation leads to a contrast enhancement signal that can be detected by ultrasonography. ${ }^{12}$ Moreover, phase change process of NDs can lead into a thermal emission. ${ }^{13)}$ Therefore, the heat generation observed by additional thermography detection can be utilized as a new platform for dual imaging modalities. In addition, the heat generation can be utilized for the treatment of tumors as well. In fact, it has been reported that the application of temperature at $39-41^{\circ} \mathrm{C}$ can cause therapeutic effects in cancer. ${ }^{14)}$ The use of gold nanoparticles or other materials such as carbon nanotubes in the tumor ablation in combination with laser irradiation has already been reported. ${ }^{15-17)}$ However, the impact of heat generation of acoustic nanodroplets in combination with low intensity ultrasound (US) on the in situ tumor regression is not clear yet. Thus, the elucidation of this function should highlight the possibility of using such nanocarriers in the cancer thermotherapy as well as in thermography and ultrasonography imaging.

The theranostic characteristics of these nanodroplets will have advantages in the treatment procedures; as droplets phase change to bubbles can be monitored by ultrasonography. Moreover, loading with anti-cancer drugs or nucleic acids can magnify their effects in combination with hyperthermia effects. For increasing the treatment efficacy and decreasing the systemic side effects, direct injection of the therapeutic compounds into tumor is also considered as a potential approach. ${ }^{18)}$ Droplets with size around $250 \mathrm{~nm}$ could be retained in the tumor tissue for a longer time after injection. ${ }^{19)}$ This approach is much possible in the case of skin superficial tumor i.e., melanoma. NDs were made with perfluropentane (PFPn) that has a boiling point of $39^{\circ} \mathrm{C}$ and hence low intensity US can be used for inducing the phase change in NDs which should not cause a serious damage to the skin barrier or the surrounding tissues.

Here we report the development of acoustic NDs made with PFPn. We investigated their physicochemical characteristics including size, and stability in vitro. Additionally, we have tested the ability of NDs in combination with US in imaging and hyperthermia therapy in B16BL6 tumor bearing mice. 


\section{MATERIALS AND METHODS}

Cells, Animals and Tumor Models Female 6-weeks-old C57BL6 mice were purchased from the Shizouka Agricultural Cooperation Association for Laboratory Animals (Shizuoka, Japan). For preparing tumor bearing mice, $1 \times 10^{6}$ of mouse melanoma cells (B16BL6) in $100 \mu \mathrm{L}$ phosphate buffered saline (PBS) were injected subcutaneously into the left flanks of mice with a 26-gauge needle. Experiments were initiated when tumors reached $5-10 \mathrm{~mm}$ in diameter after 9-14d. All experiments were approved by the Animal Experimentation Committee of the Graduate School of Pharmaceutical Sciences, Kyoto University.

NDs Fabricartion 1,2-Distearoyl-sn-glycero-3-phosphocholine (DSPC) (Avanti Polar Lipid Inc., Alabaster, AL, U.S.A.) and 1,2-distearoyl-sn-glycero-3-phosphatidylethanolamine $s$ methoxypolyethyleneglycol (PEG- 2000 -DSPE, (PEG, $M_{\mathrm{w}}=$ approx. 2000)) (NOF Co., Tokyo, Japan) in a 94:6 M ratio were dissolved in chloroform, followed by evaporation of the chloroform in a rotary evaporator, and further dried under vacuum at room temperature for overnight. The obtained lipid film was hydrated with PBS solution to get a lipid concentration of $8 \mathrm{mg} \mathrm{mL}^{-1}$ at $65^{\circ} \mathrm{C}$ for $60 \mathrm{~min}$ under mild agitation followed by sonication with bath sonicator for $3 \mathrm{~min}$ and then tip sonicator for $2 \mathrm{~min}$. For preparing droplets, $1 \mathrm{~mL}$ of liposomes was added to a $2 \mathrm{~mL}$ sterilized vial followed by adding $60 \mu \mathrm{L}$ of PFPn. The vial was capped and exposed to bath type sonication for $3-5 \mathrm{~min}$ on ice. The sample was kept at $4^{\circ} \mathrm{C}$ until further use.

Characterization of NDs The particle size and zeta potential of liposomes and NDs were determined in PBS solution $(1: 10 \mathrm{v} / \mathrm{v})$ using a Zetasizer Nano ZS instrument (Malvern Instruments Ltd., Worcestershire, U.K.). The in vitro echogenicity of NDs was evaluated as previously described. ${ }^{11)}$ Briefly, a rubber tube was placed in a water bath kept at $37^{\circ} \mathrm{C}$. A diagnostic ultrasound transducer was fixed at the edge of the rubber tube and then three echo images were taken as a background signal using an ultrasonography system (Vevo 2100, FUJIFILM VisualSonics, Toronto, Canada). After that, $10 \mu \mathrm{L}$ of NDs solution was added and mixed well with a pipette. An external ultrasound transducer was immersed in the rubber tube. Sample solution was irradiated with ultrasound at different exposure times followed by taking another three images. In vitro stability of NDs in PBS solution was evaluated according to the previous protocol. ${ }^{11)}$ One-hundred microliters of NDs were mixed with $500 \mu \mathrm{L}$ PBS and incubated in a water bath at $37^{\circ} \mathrm{C}$. Ten microliters samples were collected at different time points and analyzed with GC-MS. The retention percentage of PFPn was obtained by normalizing the peak area of PFPn at specific time to the peak area of PFPn at zero time.

In Vivo Thermography Imaging in Tumor Bearing Mice Mice were fixed on the surgical pads after conducting a proper anaesthesia with isoflurane vapor. Thermography monitor sys- tem (NEC Avio Infrared Technology Co., Tokyo, Japan) was fixed on $30 \mathrm{~cm}$ distance from the mice. Twenty-five microliters of NDs or PBS were injected in the center of the tumor area and the syringe was kept at the injection site. Five minutes later, thermographs were taken and then US irradiation was applied $\left(1 \mathrm{MHz}, 2 \mathrm{~W} . \mathrm{cm}^{-2}, 50 \%\right.$ duty, $\left.10 \mathrm{~Hz}\right)$ for $2 \mathrm{~min}$. Immediately after US exposure, thermographs were obtained again. Temperature change was estimated from the thermograph images by subtracting the background temperature. The in situ thermal distribution in the tumor was analyzed by using ImageJ software (National Institute of Health, MD, U.S.A.).

In Vivo Tumor Regression Mice were divided into three groups $(n=4-5$ each); no treatment, treatment with vehicle PBS + US, and treatment with NDs + US. After two weeks of tumor implantation, treatment was initiated. Twenty-five microliters of NDs or vehicle PBS were injected in the center of the tumor. After $5 \mathrm{~min}$, US irradiation was applied $(1 \mathrm{MHz}$, $2 \mathrm{~W} . \mathrm{cm}^{-2}, 50 \%$ duty, $10 \mathrm{~Hz}$ ) for $2 \mathrm{~min}$. Tumor volume and animal weight were determined every $2-3 \mathrm{~d}$. The tumor volume was calculated through the following formula:

$$
T=\left(L \times W^{2}\right) \times 0.5
$$

Where $T$ is the tumor volume $\left(\mathrm{mm}^{3}\right), L$ represents the long axis, and $W$ is short axis of the tumor.

Quantification of the Heat Shock Protein The heat shock protein (HSP70) was quantified for three groups $(n=3$ per group); that is, no treatment, PBS + US, and NDs + US. In this experiment, $25 \mu \mathrm{L}$ of NDs was injected to the center of the tumor, and US irradiation was applied $\left(1 \mathrm{MHz}, 2 \mathrm{~W} . \mathrm{cm}^{-2}\right.$, $50 \%$ duty, $10 \mathrm{~Hz}$ ) for $2 \mathrm{~min}$, respectively. Mice were then kept for $6 \mathrm{~h}$ at room temperature, scarified, and then tumors were removed and persevered at $-80^{\circ} \mathrm{C}$. Tumor tissues were homogenized in lysis buffer containing protease inhibitor and HSP70 level in the samples was quantified by HSP70 enzymelinked immunosorbent assay (ELISA) kit according to the maker instruction (Enzo Life Sciences Inc., NY, U.S.A.). Results were normalized by the content of protein (Dojindo Molecular Technologies, Inc., Tokyo, Japan) in each sample.

Statistical Analysis All data were analyzed as the mean \pm standard error of the mean (S.E.M.). The unpaired $t$-test and Dunnett's multiple comparison test were applied using GraphPad prism software (GraphPad Software, La Jolla, CA, U.S.A.).

\section{RESULTS AND DISCUSSION}

NDs Characteristics The precursor liposomes had an average size and zeta potential of $116.9 \pm 0.7 \mathrm{~nm}$ and $0.069 \pm 0.02 \mathrm{mV}$, respectively. After the addition of PFPn into liposomes and then sonication, NDs were produced. The average size and zeta potential of prepared NDs were $197.7 \pm 3.6 \mathrm{~nm}$ and $0.018 \pm 0.04 \mathrm{mV}$, respectively (Table 1). In order to estimate the ability of NDs to retain liquid PFPn

Table 1. Mean Particle Size of Liposomes and Nanodroplets

\begin{tabular}{lccc}
\hline \hline & Perfluorocarbon & Particle size (nm) & PDI \\
\hline Liposome (DSPC/PEG- ${ }_{2000}$-DSPE) & - & $116.9 \pm 0.7$ & $0.23 \pm 0.005$ \\
NDs (DSPC/PEG- ${ }_{2000}$-DSPE) & Perfluoropentane & $197.7 \pm 3.6$ & $0.069 \pm 0.02$ \\
\hline
\end{tabular}

$a), b)$. Data are shown as mean \pm S.E.M. $(n=3)$. 
a
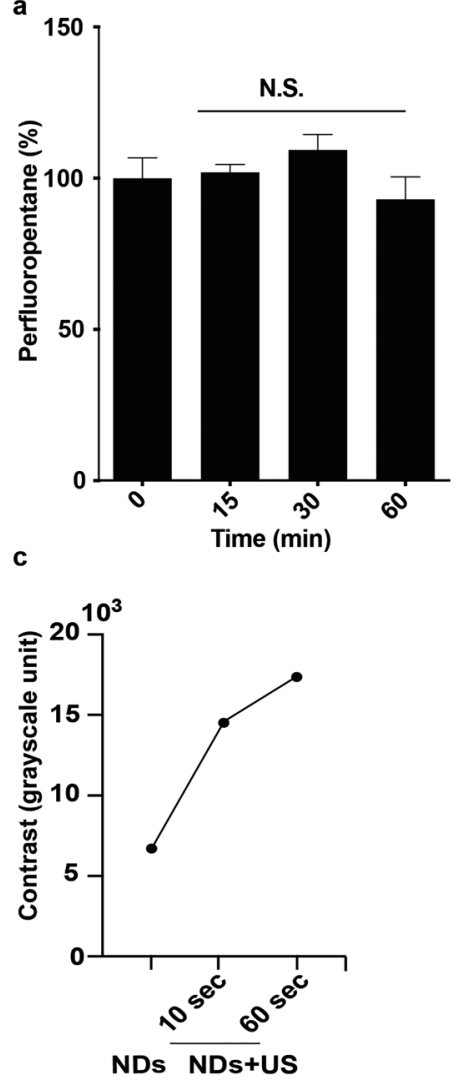

b
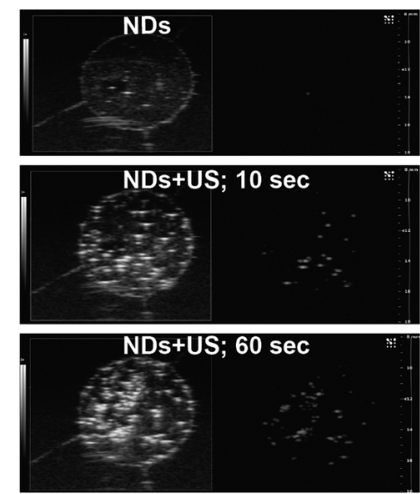

d

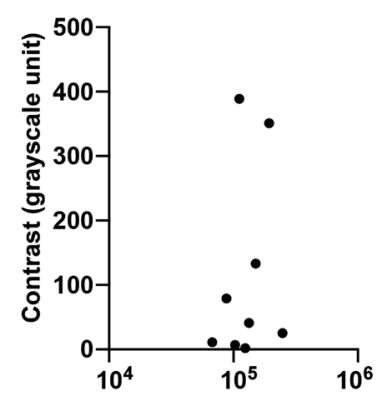

Bubbles Area ( $\left.\mu \mathrm{m}^{2}\right)$

Fig. 1. In Vitro Characteristics of NDs

a) In vitro NDs stability at $37^{\circ} \mathrm{C}$. NDs were incubated in $\mathrm{PBS}$ at $37^{\circ} \mathrm{C}$, and samples at specific time points were collected for GC-MS analysis of PFPn. b) Ultrasonography imaging of NDs activation by ultrasound irradiation in different irradiation times. Images are shown in both brightness mode (left) and contrast mode (right). c) The contrast enhancement in different irradiation time of US. d) The relationship between the contrast signal and bubbles growth area. $n=3$; mean \pm S.E.M. N.S.; (not significant) versus perfluoropentane (\%) at 0 time.

under the physiological temperature conditions, PFPn leakage from NDs in PBS at $37^{\circ} \mathrm{C}$ was determined for $60 \mathrm{~min}$ by using GCMS. As shown in Fig. 1a, there was no significant change in the PFPn amount during incubation time indicating that the use of PEG-phospholipids led to the stable retention of NDs. Ultrasonography that used for determining the phase change of NDs into bubbles has elucidated that the contrast enhancement signal was noticed with low intensity US irradiation in intensity of $2 \mathrm{~W} . \mathrm{cm}^{-2}$ for $10 \mathrm{~s}$ and the signal was further enhanced by extending the US irradiation time into $60 \mathrm{~s}$ (Figs. $1 b, c)$. These findings confirmed the phase change of PFPn in NDs from liquid phase into gas bubbles with a contrast signal that could be monitored by ultrasonography. The produced bubbles area in the in vitro setting was large enough to be visually seen, indicating the applicability of NDs in ultrasonography (Fig. 1d).

Temperature Elevation in Tumors To evaluate the thermal emission during the phase change of NDs, IR thermography was used to image the tumor bearing mice (Fig. 2a). When tumors were treated with vehicle PBS + US, there was no significant increase in temperature within the tumor. On the other hand, a significant increase in temperature was observed when tumors were treated with NDs + US (Fig. 2b), i.e., NDs + US elevated the in situ tumor temperature approximately to $39^{\circ} \mathrm{C}$ (Fig. 2c). This temperature is in the recommended range of the tumor hyperthermia. ${ }^{14)}$ Therefore, the heat emission can further be employed for the tumor hyperthermia therapy as well as tumor thermography imaging.
Furthermore, the distribution of hyperthermia area covered the tumor site where US irradiation was applied and only minor distribution of heat in the surrounding tissue indicated that the thermal emission is selectively located in the US irradiation area.

Tumor Inhibition Study and HSP70 Quantification Eight days after the tumor transplantation into the right limb of mice, tumor volume in the no treatment group quickly increased to a volume of $4020.4 \pm 1409 \mathrm{~mm}^{3}$. In mice treated with vehicle PBS + US irradiation, tumor volume was slightly reduced compared to the no treatment group $\left(3273.5 \pm 822 \mathrm{~mm}^{3}\right)$, while in the group of mice treated with NDs + US irradiation, there was significant regression of tumor volume $\left(2314.8 \pm 925 \mathrm{~mm}^{3}\right)$ (Fig. 3a). Moreover, there was no significant reduction of the animal body weight in all groups (Fig. 3b). In order to elucidate the role of the thermal emission from NDs in combination with US irradiation in tumor regression, we have studied the impact of the wellknown thermo-sensitive heat shock protein (HSP70) in the tumors. The HSP70 amount was significantly increased in tumors treated with NDs + US irradiation, comparing with the groups of no treatment and treatment with vehicle NDs only (Fig. 3c). It is known that the upregulation of HSP70 is due to a heat stress or chemical toxins. ${ }^{20}$ This machinery protein has been correlated with the upregulation of different inflammatory cytokines such as interleukin (IL)-6, tumor necrosis factor (TNF)- $\alpha$, and IL- $1 \beta$ needed for the recruitment of immune cells into tumor. ${ }^{21)}$ Additionally, the oxidative stress causes 
a

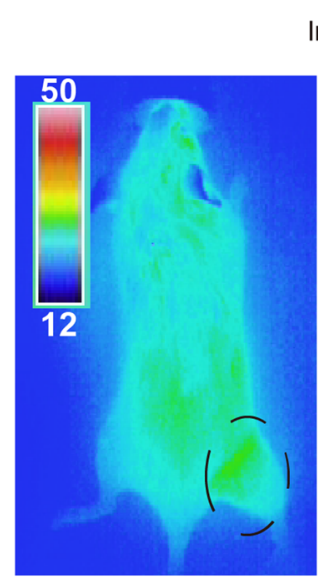

b

Intratumor injection
US irradiation
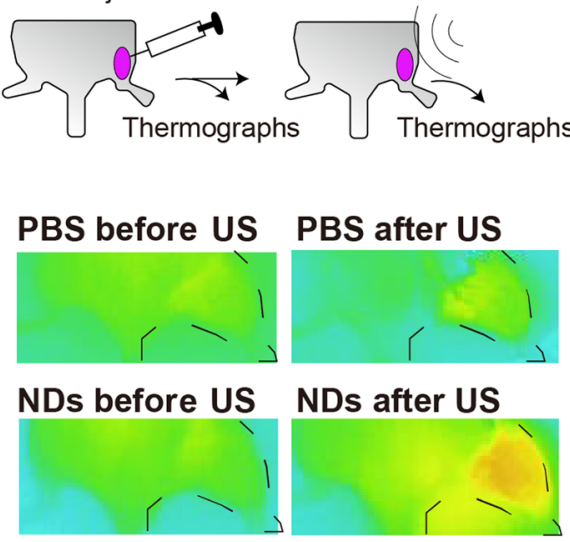

C

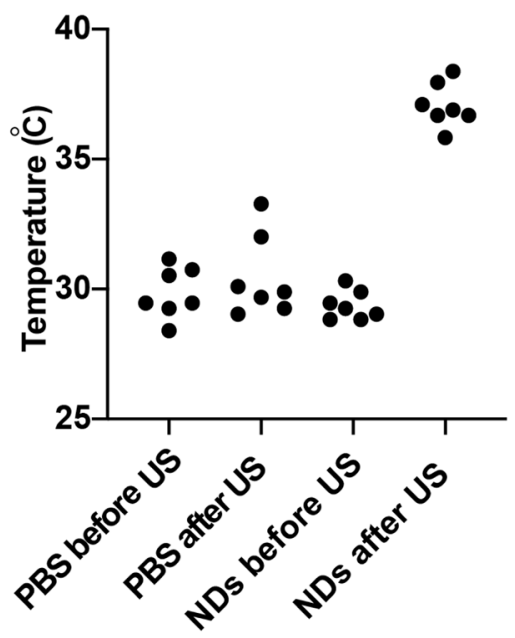

Fig. 2. In Vivo IR Thermographic Imaging

a) An overview of the IR thermography imaging in tumor bearing mice showing the overall body temperature distribution. b) Thermography images showing the temperature elevation in tumor bearing mice after intratumor injection of vehicle PBS or NDs in combination with ultrasound. c) Temperature distribution in situ in tumor. (Color figure can be accessed in the online version.)

a

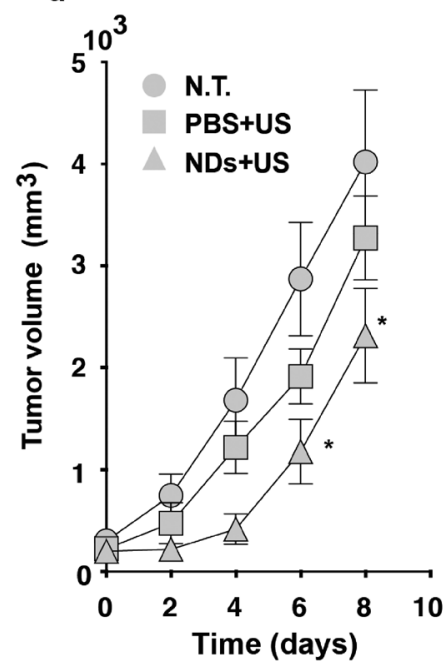

b

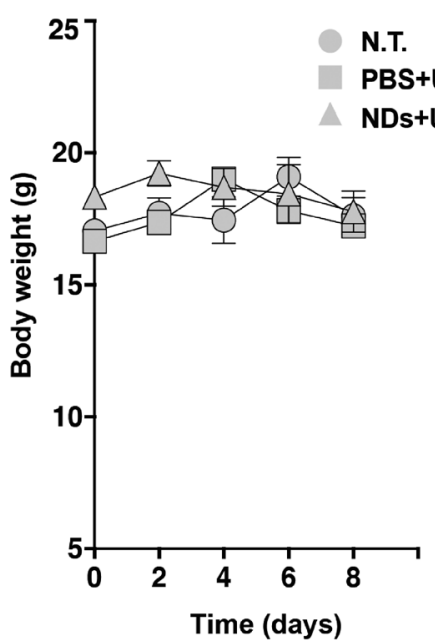

C

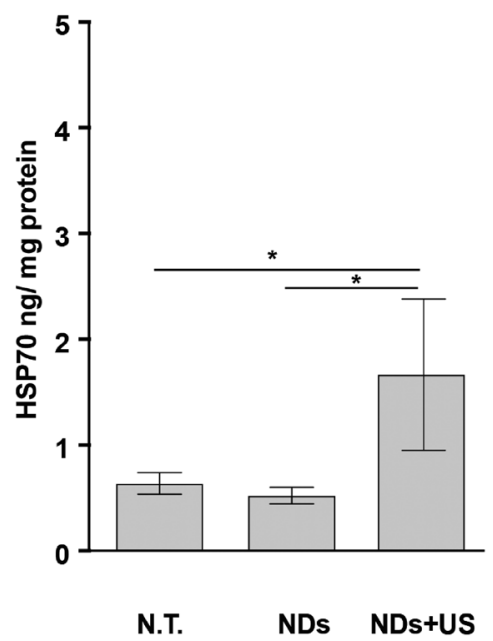

Fig. 3. Effect of NDs in Combination with US on Tumor Growth and Body Weight

a) Tumor volume in mice. b) Mice body weight. c) HSP70 quantification in tumors. Mice were divided into three groups: No treatment (N.T.); PBS in combination with US; and NDs in combination with US. Treatment was performed on the day 10 after tumor transplantation. $n=3$; mean \pm S.E.M. For HSP70 quantification, Mice were divided into three groups; no treatment (N.T.), treatment with NDs only, and treatment with NDs + US. Six hours after treatment, mice were scarified, tumors were isolated and HSP70 protein was quantified. $n=3$; the mean \pm S.E.M. $* p<0.05$.

an upregulation of HSP70 as well. ${ }^{22)}$ Although in this study we have not extensively investigated the detailed mechanism beyond the effect of NDs + US on tumor regression, we think that thermal emission from NDs during the phase change into bubbles has caused toxic events in the tumors considering the notable increase in the HSP70. The involvement of the immune cell's recruitment into the tumor site is another potential mechanism to be considered in future.

\section{CONCLUSION}

In this study, the dual theranostic abilities of phospholipidsbased PFPn NDs combined with US irradiation that can obtain thermal emission and contrast signal enhancement were investigated. The droplets phase change into bubbles was induced with an intensity of $2 \mathrm{~W} . \mathrm{cm}^{-2}$ of US in vitro in which heat emission as well as ultrasonography could be detected. The intra-tumor injection of NDs in combination with US irradiation led to a thermal emission in melanoma bearing mice and induced significant tumor regression. The intra-tumor level of HSP70 was significantly increased in tumor treated with NDs and US irradiation suggesting its participation to the therapeutic effect.

Acknowledgment This work was supported in part by a Grant-in-Aid for Scientific Research on Innovative Areas from the Ministry of Education, Culture, Sports, Science and Technology of Japan. 
Conflict of Interest The authors declare no conflict of interest.

\section{REFERENCES}

1) Ding $\mathrm{H}, \mathrm{Wu} \mathrm{F}$. Image guided biodistribution and pharmacokinetic studies of theranostics. Theranostics, 2, 1040-1053 (2012).

2) Arranja $\mathrm{A}$, Ivashchenko $\mathrm{O}$, Denkova $\mathrm{AG}$, Morawska $\mathrm{K}$, van Vlierberghe S, Dubruel P, Waton G, Beekman FJ, Schosseler F, Mendes E. SPECT/CT imaging of pluronic nanocarriers with varying poly(ethylene oxide) block length and aggregation state. Mol. Pharm., 13, 1158-1165 (2016).

3) Martin KH, Dayton PA. Current status and prospects for microbubbles in ultrasound theranostics. Wiley Interdiscip. Rev. Nanomedicine Nanobiotechnology, 5, 329-345 (2013).

4) Roland D. Theranostics health: personalized therapy and diagnosis today. Per. Med., 4, 439-444 (2007).

5) Walkey CD, Chan WCW. Quantum Dots for Traceable Therapeutic Delivery. Cancer Theranostics. Elsevier, the Netherlands, pp. 393-417 (2014).

6) Abdalkader R, Kawakami S, Unga J, Suzuki R, Maruyama K, Yamashita F, Hashida M. Evaluation of the potential of doxorubicin loaded microbubbles as a theranostic modality using a murine tumor model. Acta Biomater., 19, 112-118 (2015).

7) Tinkov S, Coester C, Serba S, Geis NA, Katus HA, Winter G, Bekeredjian R. New doxorubicin-loaded phospholipid microbubbles for targeted tumor therapy: in-vivo characterization. J. Control. Release, 148, 368-372 (2010).

8) Abdalkader R, Kawakami S, Unga J, Higuchi Y, Suzuki R, Maruyama K, Yamashita F, Hashida M. The development of mechanically formed stable nanobubbles intended for sonoporationmediated gene transfection. Drug Deliv., 24, 320-327 (2017).

9) Suzuki R, Namai E, Oda Y, Nishiie N, Otake S, Koshima R, Hirata K, Taira Y, Utoguchi N, Negishi Y, Nakagawa S, Maruyama $\mathrm{K}$. Cancer gene therapy by IL-12 gene delivery using liposomal bubbles and tumoral ultrasound exposure. J. Control. Release, 142, 245-250 (2010).

10) Rapoport N, Nam K-H. Droplet-to-bubble transition in phase-shift nanoemulsions for tumor chemotherapy. Int. J. Transp. Phenom., 12, 51-62 (2011).

11) Abdalkader R, Unga J, Yamashita F, Maruyama K, Hashida M. Evaluation of the theranostic potential of perfluorohexane-based acoustic nanodroplets. Biol. Pharm. Bull., 42, 2038-2044 (2019).

12) Sheeran PS, Dayton PA. Improving the performance of phasechange perfluorocarbon droplets for medical ultrasonography: current progress, challenges, and prospects. Scientifica, 2014, 579684 (2014).

13) Phillips LC, Puett C, Sheeran PS, Wilson Miller G, Matsunaga TO, Dayton PA. Phase-shift perfluorocarbon agents enhance high intensity focused ultrasound thermal delivery with reduced near-field heating. J. Acoust. Soc. Am., 134, 1473-1482 (2013).

14) Dewhirst MW, Vujaskovic Z, Jones E, Thrall D. Re-setting the biologic rationale for thermal therapy. Int. J. Hyperthermia, 21, 779-790 (2005)

15) Huff TB, Tong L, Zhao Y, Hansen MN, Cheng J-X, Wei A. Hyperthermic effects of gold nanorods on tumor cells. Nanomedicine, 2 125-132 (2007)

16) Murakami T, Nakatsuji H, Inada M, Matoba $Y$, Umeyama T, Tsujimoto M, Isoda S, Hashida M, Imahori H. Photodynamic and photothermal effects of semiconducting and metallic-enriched singlewalled carbon nanotubes. J. Am. Chem. Soc., 134, 17862-17865 (2012).

17) Hashida Y, Tanaka H, Zhou S, Kawakami S, Yamashita F, Murakami T, Umeyama T, Imahori H, Hashida M. Photothermal ablation of tumor cells using a single-walled carbon nanotube-peptide composite. J. Control. Release, 173, 59-66 (2014).

18) Hashida M, Kawakami S, Yamashita F. Lipid carrier systems for targeted drug and gene delivery. Chem. Pharm. Bull., 53, 871-880 (2005).

19) Nomura T, Koreeda N, Yamashita F, Takakura Y, Hashida M. Effect of particle size and charge on the disposition of lipid carriers after intratumoral injection into tissue-isolated tumors. Pharm. Res., 15, 128-132 (1998)

20) Mayer MP, Bukau B. Hsp70 chaperones: Cellular functions and molecular mechanism. Cell. Mol. Life Sci., 62, 670-684 (2005).

21) Asea A, Kraeft S-K, Kurt-Jones EA, Stevenson MA, Chen LB, Finberg RW, Koo GC, Calderwood SK. HSP70 stimulates cytokine production through a CD14-dependant pathway, demonstrating its dual role as a chaperone and cytokine. Nat. Med., 6, 435-442 (2000).

22) El Golli-Bennour E, Bacha H. Hsp70 expression as biomarkers of oxidative stress: Mycotoxins' exploration. Toxicology, 287, 1-7 (2011). 